University of Nebraska - Lincoln

DigitalCommons@University of Nebraska - Lincoln

April 1977

\title{
Theory and applications of an atoms in molecules approach to the X $\boldsymbol{\alpha}$-SCF method
}

\author{
J. Tylicki \\ University of Nebraska - Lincoln \\ R.J. Hood \\ University of Nebraska - Lincoln \\ Gordon A. Gallup \\ UNL,ggallup1@unl.edu \\ C.J. Eckhardt \\ University of Nebraska - Lincoln
}

Follow this and additional works at: https://digitalcommons.unl.edu/physicsgallup

Part of the Physics Commons

Tylicki, J.; Hood, R.J.; Gallup, Gordon A.; and Eckhardt, C.J., "Theory and applications of an atoms in molecules approach to the X $\alpha$-SCF method" (1977). Gordon Gallup Publications. 15.

https://digitalcommons.unl.edu/physicsgallup/15

This Article is brought to you for free and open access by the Research Papers in Physics and Astronomy at DigitalCommons@University of Nebraska - Lincoln. It has been accepted for inclusion in Gordon Gallup Publications by an authorized administrator of DigitalCommons@University of Nebraska - Lincoln. 


\title{
Theory and applications of an atoms in molecules approach to the $X_{\alpha}$-SCF method ${ }^{*}$
}

\author{
J. Tylicki, ${ }^{\dagger}$ R. J. Hood, ${ }^{\ddagger}$ G. A. Gallup, and C. J. Eckhardt \\ Department of Chemistry, University of Nebraska, Lincoln, Nebraska 68588 \\ (Received 29 July 1976)
}

\begin{abstract}
We have studied the $X \alpha$-SCF problem with an atoms in molecules approach. LCAO-molecular orbitals are used and the molecular charge and exchange densities are built up from atomic contributions. We have applied our method to $\mathrm{CH}_{3} \mathrm{~F}$ with subsequent comparison to $a b$ initio calculations. The $X a$-SCF dipole moment of $\mathrm{CH}_{3} \mathrm{~F}$ is $1.76 \mathrm{D}$ compared with an experimental value of $1.79 \mathrm{D}$. We also give calculations of TCNQ and TTF with comparisons to recent $X \propto$ calculations using the overlapping sphere modification of a muffin-tin potential. Quadrupole moments for TCNQ and TTF have also been determined.
\end{abstract}

\section{INTRODUCTION}

The correct treatment of exchange in $a b$ initio calculations of electronic structure presents difficulties which become exceedingly severe in large systems. These difficulties stem in large part from the inherently nonlocal character of the exchange operator. The electron gas exchange approximation or $X \alpha$ method $^{1}$ has been proposed to provide an approximate, more easily handled operator of local character for the exchange effect.

Johnson and co-workers ${ }^{2}$ have combined the $X \alpha$ method with the $\mathrm{KKR}^{3}$ multiple scattered wave procedure to do electronic structure calculations. Ellis and co-workers ${ }^{4}$ have devised a numerical procedure involving Diophantine integration, and more recently Sambe and Felton ${ }^{5}$ have investigated the structures of some small molecules using LCAO-MO-X $\alpha-S C F$ procedure.

The present article describes an LCAO-MO-X $\alpha-S C F$ technique which is designed with two specific goals in mind:

(a) The efficient handling of large systems;

(b) The duplication, insofar as possible, of the $a b$ initio values of occupied orbital energies.

In order to implement the first of these we use an atoms in molecules approximation to the electronic charge and exchange densities, by building up the molecular quantities as sums of atomic contributions.

The second goal is accomplished by starting with the $a b$ initio Hartree-Fock equations and applying the various approximations at this stage.

In the next section we describe our procedure. This is followed by sections giving some approximations for atomic charge and exchange densities and applications of our method to $\mathrm{CH}_{3} \mathrm{~F}$, TCNQ (tetracyano-p-quinodimethane) and TTF (bis-tetrathiofulvalene).

\section{MATHEMATICAL DETAILS}

We use as a starting point the Hartree-Fock Equations for the system,

$$
\begin{aligned}
H u_{k}= & -\frac{1}{2} \nabla^{2} u_{k}(1)-\sum \frac{Z_{Y}}{r_{\gamma}} u_{k}(1)+2 \int \sum_{l}\left|u_{l}(2)\right|^{2} \frac{d \tau_{2}}{r_{12}} u_{k}(1) \\
& -\sum_{l} u_{l}(1) \int u_{l}^{*}(2) u_{k}(2) \frac{d \tau_{2}}{r_{12}}
\end{aligned}
$$

$$
=\epsilon_{k} u_{k} \text {. }
$$

The bulk of the labor of $a b$ initio calculations lies with the Coulomb and exchange terms of the Fock operator, and it is clear that the development of effective approximations for these terms would bring the calculation of large systems into an easily accessible region. We emphasize that approximations are needed for both the Coulomb and exchange terms to produce the desired decrease in labor of the calculations.

Our atoms in molecules approach consists of writing the charge density, $\rho$, as a sum of atomic contributions

$$
2 \sum\left|u_{k}\right|^{2}=\rho \simeq \sum_{\gamma} \delta_{\gamma} \rho_{\gamma},
$$

where the $\rho_{y}$ are charge densities of individual atoms and the $\delta_{\gamma}$ are constants which measure the effective charge on the atom. The $\rho_{\gamma}$ are spherically symmetric approximations to the charge densities of individual atoms. We discuss these functions in the next section.

We use the standard $X \alpha$ approximation for the exchange term,

$$
\begin{aligned}
V_{X \alpha} & =-6 \alpha\left[\left(\frac{3}{8} \pi\right) \rho\right]^{1 / 3} \\
& =-6 \alpha\left(\frac{3}{8} \pi\right)^{1 / 3} \sum_{\gamma} \delta_{\gamma}^{1 / 3} \rho_{\gamma}^{1 / 3}+R,
\end{aligned}
$$

where we have replaced $\rho$ with the approximation given by (2). The remainder $R$, is added since the cube root of a sum is not equal to the sum of cube roots. The nature of $R$ is easy to deduce. It is small in any region of space where only one of the terms $\delta_{\gamma} \rho_{y}$ is significantly different from zero, or where $p$ is very small. The regions of largest $R$ occur where there is significant overlap of two or more of the $\rho_{y}$. An approximation with some analogies to semiempirical CNDO methods would result if we take $R$ to be zero everywhere. A less drastic alternative involves modifying $\rho_{\gamma}^{1 / 3}$ with an auxiliary function so as to minimize the serious effects of dropping $R$ without compensations, and we have

$$
V_{X \alpha} \simeq-6 \alpha\left(\frac{3}{8} \pi\right)^{1 / 3} \sum_{\gamma} \delta_{\gamma}^{1 / 3} \rho_{\gamma}^{1 / 3} \xi_{\gamma} .
$$

A number of function types for $\zeta_{\gamma}$ would undoubtedly work, but a convenient and satisfactory choice consists of a Gaussian function, $\zeta_{\gamma}=\exp \left(-\epsilon \gamma_{\gamma}^{2}\right)$ with $\epsilon$ the same for all atoms. The calculations described in this article use $\epsilon=0.12$, a value giving $X \alpha$ bound state orbital energies close to those from $a b$ initio calculations. The 
TABLE I. Coefficients and exponential scale factors for least square approximations to charge and exchange densities of hydrogen, carbon, nitrogen, oxygen, fluorine, neon, and sulfur. [See Eqs. (5) and (6).]

\begin{tabular}{|c|c|c|c|c|c|c|}
\hline & $k=$ & 1 & 2 & 3 & 4 & 5 \\
\hline \multirow[t]{4}{*}{$\mathrm{H}$} & $s_{k}$ & 119.9984 & 19.84331 & 5.06226 & 1. 64182 & 0.59198 \\
\hline & $a_{k}$ & 0.13632 & 0.22387 & 0.26210 & 0.17486 & 0.03226 \\
\hline & $t_{k}$ & 40.00 & 6.614 & 1.678 & 0.5472 & 0.1973 \\
\hline & $b_{k}$ & 0.09102 & 0.14018 & 0.23560 & 0.18134 & 0.27704 \\
\hline \multirow[t]{4}{*}{$\mathrm{C}$} & $s_{k}$ & 119.09696 & 27.28693 & 6.06064 & 1.60340 & 0.46932 \\
\hline & $\hat{a_{k}}$ & 26.95817 & 30.97315 & 1. 79244 & 0.05466 & 0.22263 \\
\hline & $t_{k}$ & 39.67 & 9.075 & 2.0202 & 0.5345 & 0.15644 \\
\hline & $b_{k}$ & 1. 16214 & 1.90694 & 0.09989 & 0.04348 & 0.59146 \\
\hline \multirow[t]{4}{*}{$\mathrm{N}$} & $s_{k}$ & 125.81507 & 26.97817 & 6.08744 & 1. 13328 & 0.44937 \\
\hline & $a_{k}$ & 94.71361 & 33.78108 & 0.46759 & 0.61020 & 0.12411 \\
\hline & $t_{k}$ & 42.605 & 8.993 & 2.029 & 0.3777 & 0.1498 \\
\hline & $\hat{b_{k}}$ & 2.59468 & 1.38888 & 0.02586 & 0.50809 & 0.33958 \\
\hline \multirow[t]{4}{*}{$\mathrm{O}$} & $s_{k}$ & 131.98357 & 28.04665 & 6.46593 & 2. 37572 & 0.66052 \\
\hline & $a_{k}$ & 145.90291 & 36.91763 & -1.40304 & 1.72551 & 0.37901 \\
\hline & $t_{k}$ & 43. 994 & 9.346 & 2.155 & 0.7919 & 0.2203 \\
\hline & $b_{k}$ & 3.48510 & 0.85545 & 0.09331 & 0.38982 & 0.64669 \\
\hline \multirow[t]{4}{*}{$F$} & $s_{k}$ & 135.34790 & 27.99038 & 7.10991 & 3. 75935 & 0.86597 \\
\hline & $a_{k}$ & 203.73935 & 37.80394 & -5.64042 & 4. 91189 & 0.69148 \\
\hline & $t_{k}$ & 45.118 & 9.330 & 2.370 & 1.253 & 0.2887 \\
\hline & $b_{k}$ & 4.34673 & 0.156629 & 0.49047 & 0.16314 & 0.86562 \\
\hline \multirow[t]{4}{*}{$\mathrm{Ne}$} & $s_{k}$ & 155.55228 & 27.18310 & 8.19035 & 5.63028 & 1. 18456 \\
\hline & $a_{k}$ & 290.72259 & 41.41658 & -18.21111 & 14.63973 & 1.33422 \\
\hline & $t_{k}$ & 51.851 & 9.061 & 2.730 & 1.877 & 0.3948 \\
\hline & $\hat{b}_{k}$ & 5.08178 & -0.44724 & 1.58680 & -0.69876 & 1. 14821 \\
\hline \multirow[t]{4}{*}{$\mathbf{S}$} & $s_{k}$ & 411.4861 & 13.83517 & 3.733565 & 3. 370554 & 0.4471470 \\
\hline & $a_{k}$ & 2031.984 & 39.68394 & 26.52671 & -19.36931 & 0.3922622 \\
\hline & $t_{k}$ & 140.0096 & 11.55385 & 7.579065 & 0.1758505 & 0.07150376 \\
\hline & $b_{k}$ & 4. 147832 & -1.401360 & 9.031689 & 0.5754152 & 0.1430481 \\
\hline
\end{tabular}

real convenience of a Gaussian function for $\zeta_{\gamma}$ is, of course, a result of our use of this same function type to represent $\rho$ and $\rho^{1 / 3}$. This correction produces a value for the exchange energy which is very close to the $a b$ initio. In $\mathrm{CH}_{3} \mathrm{~F}$ the $\mathrm{X} \alpha$ gives 16.04 a. $\mathrm{u}$. compared with 16.46 a. u. for $a b$ initio. These results on $\mathrm{CH}_{3} \mathrm{~F}$ are discussed more fully below.

Once the types of atoms and their positions in the system are fixed, the only variables in $\rho$ and $\rho^{1 / 3}$ are the $\delta_{\gamma}$. If the molecular orbitals for the system are known, the values of $\delta_{\gamma}$ may be determined by a standard Mulliken population analysis. This connection between $\delta_{\gamma}$ and MO's provides an iterative procedure for the $X \alpha-S C F$. An initial set of $\delta_{\gamma}$ may be guessed, from which a Fock-matrix is determined, which provides a set of orbitals from which a set of $\delta_{\gamma}$ may be calculated; the process is then carried to self-consistence. An initial guess of $\delta_{\gamma}=1$ has been satisfactory in all cases tried.

Because of our approximations, the procedural step in which the Fock matrix is evaluated may be executed without the extensive tensorial contraction operations characteristic of $a b$ initio LCAO-MO methods. The number of integrals for the atoms in molecules X $\alpha$-SCF is much smaller than that for $a b$ initio methods. Thus, we must evaluate integrals for the overlap kinetic energy, Coulomb interaction, charge density and the ex- change potential. A system comprising $N_{A}$ atoms to be studied with a basis of $N_{0}$ AO's requires the evaluation and storage of $\left(2 N_{A}+3\right) N_{0}\left(N_{0}+1\right) / 2$ integrals. These need be calculated only once, since the SCF steps involve the manipulation of the $\delta_{\gamma}$ and the MO's only. The small number of integrals and the simplicity of the SCF step are the reasons for the rapidity of our atoms in molecules $X \alpha-S C F$ method. (AIM-X $\alpha-S C F$ ).

\section{GAUSSIAN APPROXIMATION TO $\rho_{\gamma}$ AND $\rho_{\gamma}^{1 / 3}$}

The $X \alpha-S C F$ calculation procedure outlined in the previous section depends crucially upon having adequate approximations for atomic charge and exchange densities. In all cases $\rho_{\gamma}$ and $\rho_{\gamma}^{1 / 3}$ are represented as sums of spherical Gaussians:

$$
\begin{aligned}
& \rho_{\gamma}=\sum_{k} a_{k} \exp \left(-s_{k} r_{\gamma}^{2}\right) ; \\
& \rho_{\gamma}^{1 / 3}=\sum_{k} b_{k} \exp \left(-t_{k} r_{\gamma}^{2}\right) .
\end{aligned}
$$

The constants $a_{k}, s_{k}, b_{k}$, and $t_{k}$ in (5) and (6) may be determined by a least squares fit of $\rho_{\gamma}$ to spherically averaged $a b$ initio atomic charge density functions, followed by another least squares fit of $\left(\rho_{\gamma}^{1 / 3}\right)^{3}$ to $\rho_{\gamma}$. This has been done for $\mathrm{H}$, the second row atoms, $C, N, O$, and $F$, and the third row atom, S. Five term approximations for $\rho_{\gamma}$ and $\rho_{\gamma}^{1 / 3}$ are given in Table I for each of 


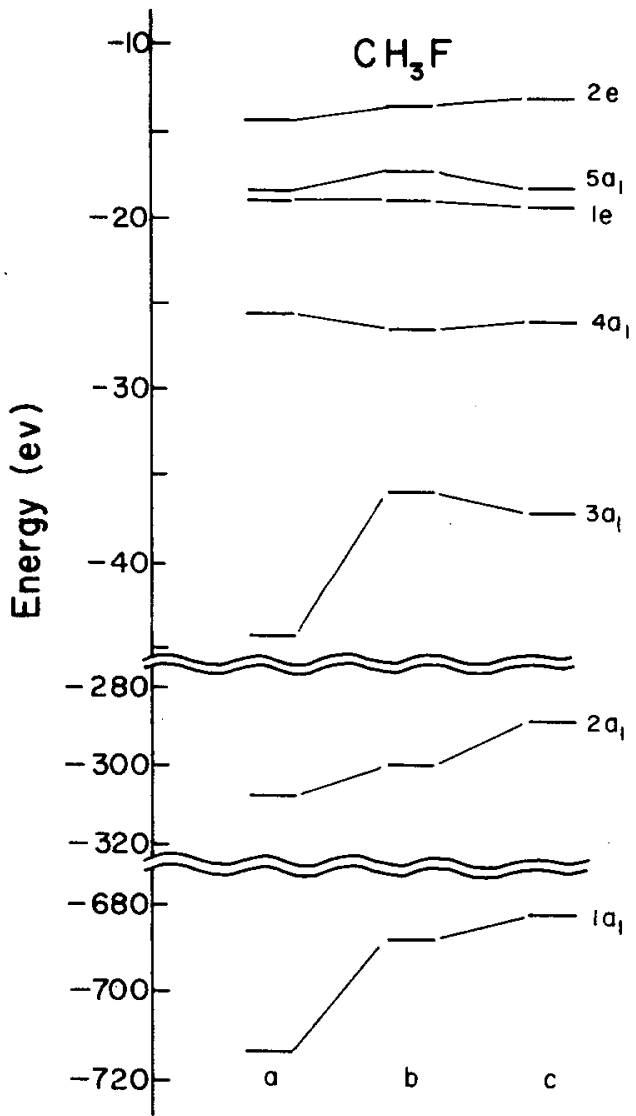

FIG. 1. Comparison of orbital energies for various calculations of the $\mathrm{CH}_{3} \mathrm{~F}$ molecule: (a) $\mathrm{Ab}$ initio results, (b) $\chi \alpha-\mathrm{SCF}$ with Whitten basis, and (c) $\chi_{\alpha}-\mathrm{SCF}$ with $3 \mathrm{G}-\mathrm{STO}$ basis.

these atoms. These are in atomic units so that the integral of $\rho_{\gamma}$ is just the atomic number.

Approximations equivalent to those in Table $I$ are in principle obtainable for every element and studies are underway to obtain approximations to $\rho$ and $\rho^{1 / 3}$ for many other atoms.

\section{SPECIFIC CALCULATIONS USING THE ATOMS IN MOLECULES $X \alpha$-SCF METHOD}

\section{A. Methyl fluoride}

The orbital energies and electric dipole moment of $\mathrm{CH}_{3} \mathrm{~F}$ have been determined using two different $\mathrm{AO}$ bases:

(a) The Gaussian lobe basis given by Whitten; ${ }^{6}$

(b) The 3G-STO basis and scale factors given by Pople and co-workers. ${ }^{7}$

The atomic charge and exchange densities from Table I were used. Figure 1 shows a comparison of the orbital energies of our two $\mathrm{CH}_{3} \mathrm{~F}$ calculations with $a b$ initio results obtained with the (b) basis above. The AIM-X $\alpha-$ SCF reproduces the $a b$ initio energies well and the relatively small differences resulting from the basis change also show our method to have satisfactory stability. This is also shown by the values of the dipole moment: Gaussian lobe, 1.76 D; 3G-STO, 1.79 D. There is very satisfactory agreement of both of these with the experimental value of $1.79 \mathrm{D} .^{8}$ The $a b$ initio dipole moment of 1.48 D for basis (b) is rather poor. The exact agreement of the 3G-STO calculations cannot be considered more than fortuitous.

\section{B. Tetracyanoquinodimethane}

TCNQ is the acceptor molecule in several organic electron-donor-acceptor crystals which show electrical conductivity and its properties are of considerable interest. In addition, the electronic structure of TCNQ has been obtained recently with the overlapping sphere modification of the $X \alpha$-scattered wave method. ${ }^{8}$ We have calculated TCNQ to provide a comparison of our results with those from the modified muffin-tin potential.

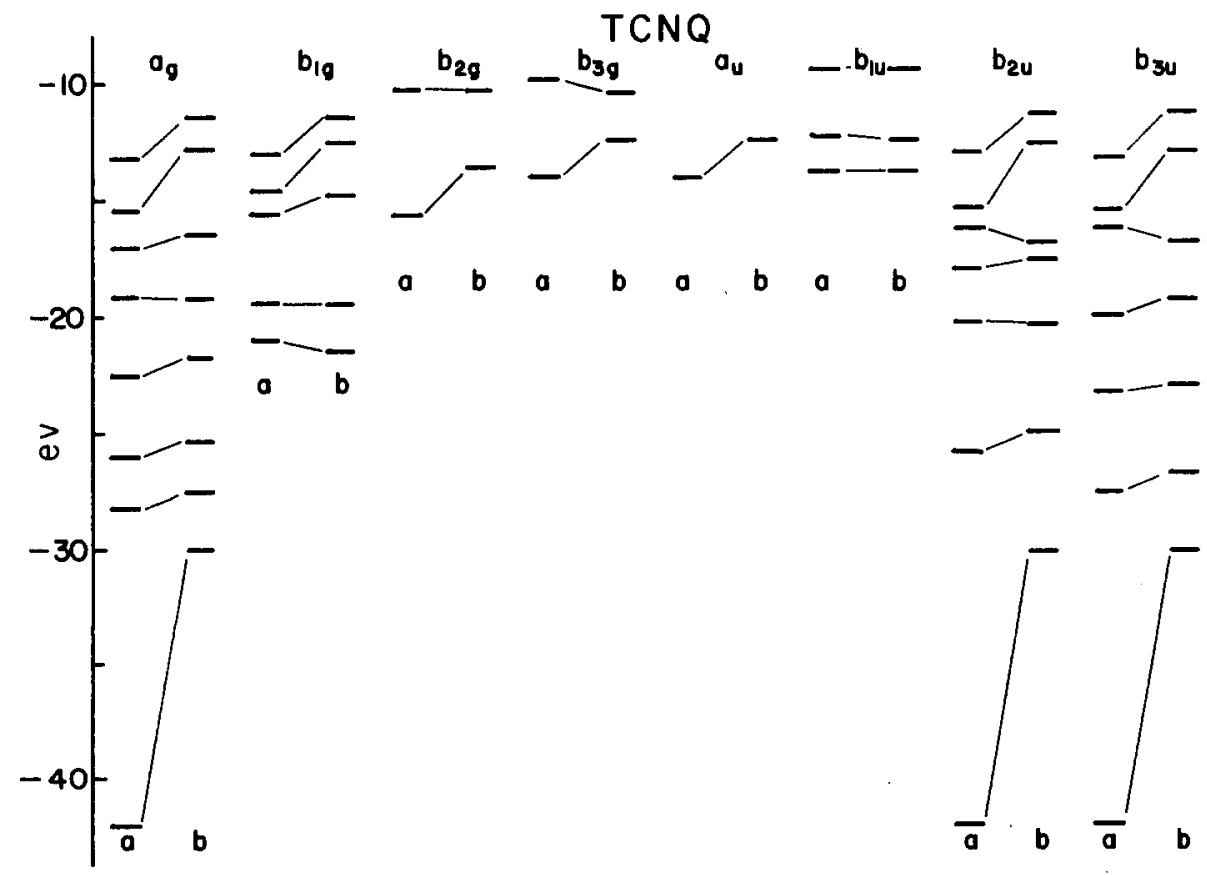

FIG. 2. Comparison of orbital energies of TCNQ for AIM$\chi \alpha-\mathrm{SCF}$ approach and modified muffin-tin potential; (a) Ref. 9 and $(b)$ this work. 


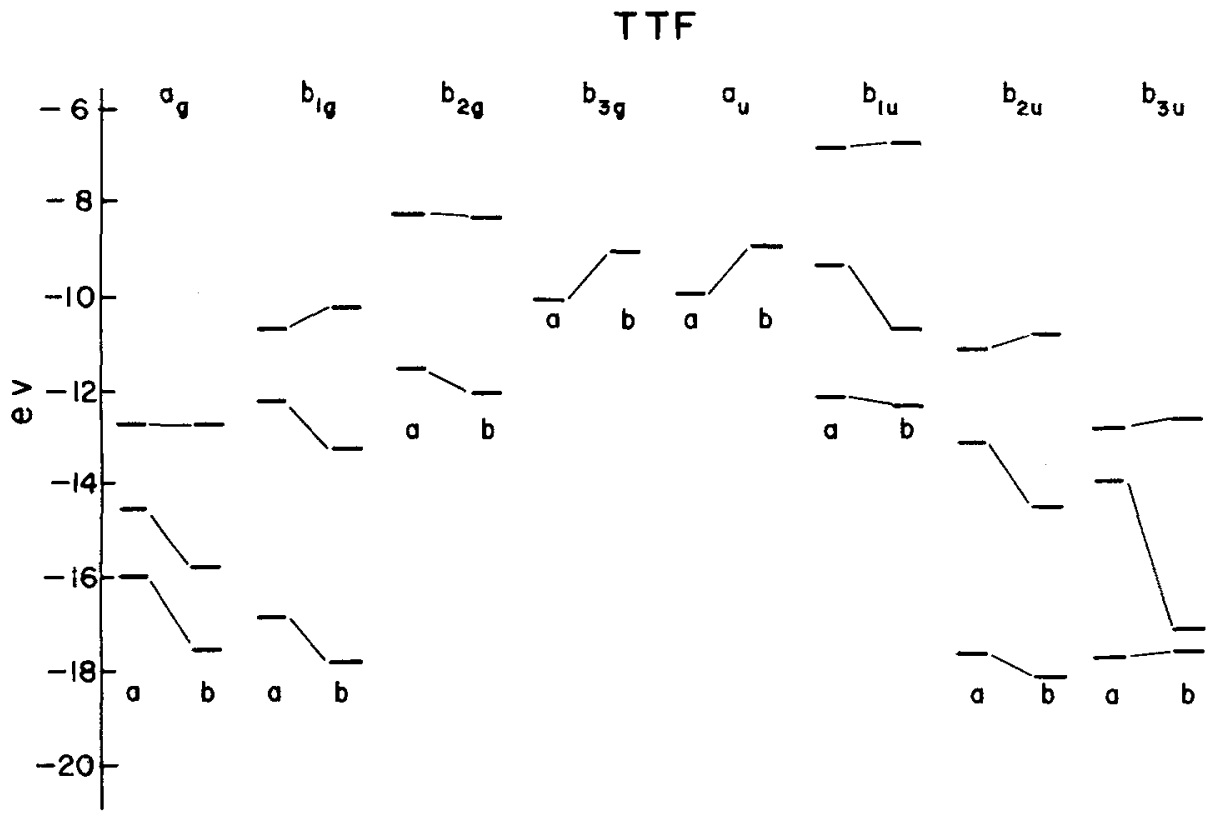

FIG. 3. Comparison of orbital energies of TTF for AIM- $\chi_{\alpha-}$ SCF approach and modified muffin-tin potential: (a) Ref. 10 and (b) this work.
The functions given in Table I provide the necessary atomic densities to do the calculations and the 3G-STO's are used for the AO basis. We show our valence orbital energies and those of Herman, Williams, and Johnson $^{9}$ (Model IV), in Fig. 2. We see that the orbital energies from the two calculations are qualitatively very similar although there are quantitative differences. These differences are reflected most strongly in the region of CN group orbitals at the bottom of the diagram. The positions of these were reported to be very sensitive to the differing overlapping sphere models. ${ }^{9}$

\section{Tetrathiofulvalene}

TTF is the second component of the most familiar TCNQ charge transfer crystal. We have calculated the electronic structure of TTF and our valence orbital energies are compared with those of Bennett and Her$\operatorname{man}^{10}$ in Fig. 3. The latter workers also used the overlapping sphere approximation in this determination. Our calculation was again done with a 3G-STO basis. The sulfur orbital exponential scale factors were obtained from the single- $\zeta$ SCF results of Clementi and Roetti. ${ }^{11}$

We see that the two computation methods again predict a similar qualitative structure for the valence orbital ene rgies but with some quantitative differences. The differences are similar to those in the upper part of Fig. 2. Although TTF and TCNQ have the same total

TABLE II. Quadrupole moments (esu $\mathrm{cm}^{2}$ ) of TCNQ and TTF.

\begin{tabular}{lrr}
\hline & \multicolumn{1}{c}{ TCNQ } & \multicolumn{1}{c}{ TTF } \\
\hline$Q_{x x}$ & $4.80 \times 10^{-25}$ & $0.93 \times 10^{-25}$ \\
$Q_{y y}$ & $-0.74 \times 10^{-25}$ & $-4.58 \times 10^{-25}$ \\
$Q_{s z}$ & $-4.06 \times 10^{-25}$ & $3.65 \times 10^{-25}$ \\
\hline \hline
\end{tabular}

number of electrons, TTF has significantly fewer valence electrons because of the sulfur.

\section{Quadrupole moments}

Recent results on the $a b$ initio calculation of interaction potential of two hydrogen molecules indicates that the influence of these small molecular electric quadrupoles can be as important as dispersion interactions. ${ }^{12}$ Thus, the ability to easily calculate quadrupole moments for systems such as TTF and TCNQ from reasonable wavefunctions may be a useful feature of any theoretical method. The quadrupole moments of both TCNQ and TTF are given in Table II. The values here are quite sizeable and indicate that consideration of quadrupole interactions may be necessary to the understanding of electronic interactions and structures of complexes of these molecules.

\section{CONCLUSIONS}

The AIM-X $\alpha$-SC F method promises to be a useful calculative procedure for determining the electronic properties of large molecules. A particular benefit of this approach is that the method uses analytical functions in all steps of the procedure. Finally, MO's which are linear combinations of Gaussians or STO's are obtained. This greatly facilitates efficient calculations for large systems and alleviates the sensitivity of numerical $X \alpha$ calculations to the choice of the overlapping sphere. The results of calculations on the large systems, TTF and TCNQ, are in agreement with reported overlapping sphere $X \alpha$-scattering calculations.

\section{ACKNOWLEDGMENTS}

The authors wish to express their appreciation to Professor Clyde Edmiston for useful conversations and to the University of Nebraska Computing Network for the computational facilities to do this work. 
* Supported by the National Science Foundation through Grant DMR75-01058, and the Research Council, University of Nebraska.

$\dagger$ Present Address: Department of Physics, University of Indiana, Northwest, Gary, Indiana 48304.

$\$$ Permanent Address: Department of Chemistry, Marygrove College, Detroit, Michigan 48221.

${ }^{1}$ J. C. Slater, J. Chem. Phys. 43, 8228 (1965); Adv. Quantum Chem. 6, 1 (1972).

${ }^{2}$ K. H. Johnson, J. Chem. Phys. 45, 3085 (1966); Int. J. Quantum Chem. Symp. 1, 361 (1967); 2, 233 (1968); 4, 153 (1971); K. H. Johnson and F. C. Smith, Phys. Rev. Lett. 24, 139 (1970); Chem. Phys. Lett. 7, 541 (1970).

${ }^{3}$ J. Koringa, Physica 13, 392 (1947); W. Kohn and N. Rostoker, Phys. Rev, 94, 1111 (1954).

${ }^{4}$ D. E. Ellis and G. S. Painter, Phys. Rev. B 2, 2887 (1970);
Ibid. 1, 4747 (1970): E. J. Baerends, D. E. Ellis, and P. Ros, Chem. Phys. 2, 41 (1973).

${ }^{5}$ H. Sambe and R. H. Felton, J. Chem. Phys. 61, 3862 (1974); Ibid. 62, 1122 (1975)

${ }^{6} \mathrm{~J}$. L. Whitten, J. Chem. Phys. 44, 359 (1966).

${ }^{7}$ W. J. Hehre, R. F. Stewart, and J. A. Pople, J. Chem. Phys. 51, 2657 (1969).

${ }^{8} \mathrm{~W}$. Gordy, W. V. Smith, and R. Trambarulo, Microwave Spectroscopy (Wiley, New York, 1953), p. 361.

${ }^{9}$ F. Herman, A. R. Williams, and K. H. Johnson, J. Chem. Phys. 61, 3508 (1974).

${ }^{10}$ B. I. Bennett and F. Herman, Phys. Rev. Lett. (to be published).

${ }^{11}$ E. Clementi and C. Roetti, At. Data Nucl. Data Tables 14, 177 (1974).

${ }^{12} \mathrm{G}$. A. Gallup, J. Chem. Phys. 66, 2252 (1977). 